\title{
Critical points in coupled Potts models and critical phases in coupled loop models
}

\author{
Paul Fendley ${ }^{1,2}$ and Jesper L. Jacobsen ${ }^{3,4}$ \\ ${ }^{1}$ All Souls College and the Rudolf Peierls Centre for Theoretical Physics, \\ University of Oxford, 1 Keble Road, OX1 3NP, UK \\ ${ }^{2}$ Department of Physics, University of Virginia, Charlottesville, VA 22904-4714 USA \\ ${ }^{3}$ Université Pierre et Marie Curie, 4 place Jussieu, 75252 Paris Cedex 05, France \\ ${ }^{4}$ Institut de Physique Théorique, CEA Saclay, 91191 Gif-sur-Yvette, France
}

November 14, 2018

\begin{abstract}
We show how to couple two critical $Q$-state Potts models to yield a new self-dual critical point. We also present strong evidence of a dense critical phase near this critical point when the Potts models are defined in their completely packed loop representations. In the continuum limit, the new critical point is described by an $S U(2)$ coset conformal field theory, while in this limit of the the critical phase, the two loop models decouple. Using a combination of exact results and numerics, we also obtain the phase diagram in the presence of vacancies. We generalize these results to coupling two Potts models at different $Q$.
\end{abstract}

\section{Introduction}

The $Q$-state Potts model is of fundamental interest in two-dimensional statistical physics. It arises in an amazing number of different contexts in mathematics and theoretical physics alike: algebra, combinatorics, probability theory, graph theory, integrability, conformal field theory (CFT), stochastic Loewner evolution (SLE), etc. Thus progress in understanding the Potts model serves as a useful gauge for progress in many of these fields.

Via its underlying algebraic formulation - in terms of the Temperley-Lieb algebra [1 - the Potts model can be recast in several similar ways, but which do however present subtle differences. Its formulation as a model of completely packed loops with non-local weights is prototypical within the large (and still growing) class of so-called loop models in classical statistical mechanics. Such models push the limits of CFT, as they present non-unitary and logarithmic features [2], and they link directly to physics-inspired models of extended objects, such as polymers. The loops underlying the Potts model role as convenient lattice regularizations of SLE traces [3]. They can also be interpreted as particle trajectories in various models of disordered systems, such as the spin quantum Hall effect [4].

Studying several coupled Potts models is a challenge for all of the techniques listed above, and also serves as a worthwhile purpose on its own right.

In particular, coupled Potts models arise naturally when studying quantum loop models [5, 6]. Each configuration in the classical loop model is a basis element of the Hilbert space of the quantum loop model. The reason why two coupled copies of the loop model arise in this context 
is elementary quantum mechanics. When computing expectation values, one of course weights by the wavefunction squared. Thus one loop model comes from the bra, and the other from the ket. Our results are very useful for quantum loop models, because the critical phase we find below in the classical model implies the existence of a phase with topological order in the quantum loop model with completely packed loops [7, 5, 6. In a topological phase, the excitations are fractionalized, and have anyonic statistics. These features are possible when the ground state of the quantum system is a sum over different loop configurations; the non-locality of loops makes anyonic braiding possible. For the anyonic excitations to be deconfined, the underlying classical loop model must be critical, so that all lengths of loops appear in the partition function.

Coupled Potts models are also at play when studying a single Potts model with quenched random bond disorder. Using the replica trick, this corresponds to coupling $\mathcal{N}$ pure Potts models via the product of their energy operators, followed by the formal limit $\mathcal{N} \rightarrow 0$. For $Q-2 \ll$ 1 and in the limit of weak non-frustrated disorder, this can be done within the perturbative renormalization group (RG) approach of CFT [8]. There exists ample numerical evidence that the replica symmetry is not broken in this case. Moreover, the formal expressions obtained for the critical exponents corresponding to $\mathcal{N}>2$ integer have been identified - order by order in the perturbation - with those of particular critical points of $\mathcal{N}$ coupled lattice models. If exact results for these critical points could be obtained exactly, their analytical continuation to the $\mathcal{N} \rightarrow 0$ limit would provide exact results for the random-bond Potts model [9]. In this context, the case of two coupled models to be studied here is somewhat particular, since the RG flows depend on the coupling $g$ in the combination $(2-\mathcal{N}) g$.

In the present study we assume (as in [9]) that the coupled Potts models are self-dual, i.e., they are left invariant under the combined duality transformation of both underlying models. Although it is known in the special case of $Q=2$ that mutually dual pairs of critical points do exist, it seems plausible that self-duality will generically allow for the highest multicritical — and thus most interesting-behavior.

Among the directions that we leave for future investigation, one of the most elusive is undoubtedly to provide a Coulomb Gas (CG) description for coupled Potts models. A putative CG construction would allow one to compute the critical exponents of certain non-local operators analytically, an issue that we only pursue numerically here. Suffice it to say that our analytical result for the central charge at the new self-dual critical point hints at a CG with three height components, but only two of those are easy to identify geometrically. This is not without reminding us of Ref. [10].

The plan of the paper is as follows. In section 2 we introduce the models to be studied, and summarize our main results. In section 3 we derive the location and universality class of a new self-dual critical point. The cornerstone of the arguments is a level-rank duality in a related $S O(N)$ symmetric integrable model. Numerical calculations are used to verify the consistency of the proposed phase diagram, and to give results for the dimensions of watermelon operators at the new critical point. In section 4 we argue that adjacent to the new critical point, the loop model possesses a critical phase, with parameter-independent critical exponents. We provide evidence that the phase transition into this phase is simultaneously first and second order. In section 5 we generalize the model to include vacancies. We argue that dilution is an irrelevant perturbation of the completely packed model. The phase diagram for the dilute model is presented. Finally, in section 6] we generalize our results to the case where the two coupled Potts model have a different number of states. 


\section{The models and their phase diagrams}

The original formulation of the $Q$-state Potts model places a spin $\sigma_{i}$ taking integer values $1,2, \ldots, Q$ on each site of some lattice. In this paper we shall discuss exclusively the square lattice, but we expect by universality that our results will apply qualitatively and sometimes quantitatively to other lattices. The Potts model has nearest-neighbor interactions invariant under the permutation group $S_{Q}$, so that the interaction energy $J \delta_{\sigma_{i}, \sigma_{j}}$ only depends on whether adjacent spins $\sigma_{i}$ and $\sigma_{j}$ are the same or different.

The Potts model on the square lattice has a Kramers-Wannier (high- to low-temperature) duality [11]. This is a non-local map of spins which preserves the bulk free energy. The self-dual point is critical [12] when $Q \leq 4$. In a field-theory description of the region around these critical points, taking the temperature off the self-dual value corresponds to perturbing by the energy operator $\varepsilon$ (the continuum limit of $J \delta_{\sigma_{i}, \sigma_{j}}$ ). The energy operator is odd under duality, $\varepsilon \rightarrow-\varepsilon$, so that perturbing by this operator with one sign describes the high-temperature phase, while the other sign gives the low-temperature phase.

Although the original definition of the Potts model requires $Q$ to be an integer, there exist generalizations to all $Q$ which are still commonly known as the Potts model. We will treat two closely-related but distinct models, which we refer to as the loop and the height models. The transfer matrices of the original Potts model and the two generalizations are most conveniently defined in terms of the Temperley-Lieb algebra; each model then corresponds to a different representation. The algebra for a system of width $L$ has $L$ generators $e_{i}$ acting at positions $i=1,2, \ldots, L$ as well as the identity $I$, which obey the relations [1]

$$
e_{i}^{2}=\sqrt{Q} e_{i}, \quad e_{i} e_{i \pm 1} e_{i}=e_{i}, \quad e_{i} e_{j}=e_{j} e_{i} \text { for }|i-j|>1
$$

The transfer matrix at the self-dual isotropic point for width $L$ is then

$$
T=R_{1} R_{3} \ldots R_{L-1} R_{2} R_{4} \ldots R_{L}
$$

where $R_{i}=I+e_{i}$ for the ferromagnetic self-dual point, and $R=I-e_{i}$ for the (non-unitary) antiferromagnetic self-dual point. The partition function for an $M \times L$ lattice with periodic boundary conditions in the "time" direction is then given by

$$
Z=\operatorname{tr} T^{M} .
$$

In this formulation, duality amounts to exchanging $e_{i}$ with $I$ on each plaquette.

It is convenient to parameterize

$$
\sqrt{Q}=2 \cos \left(\frac{\pi}{k+2}\right)
$$

defining the parameter $k$. When $k$ is an integer obeying $k \geq 2$ (so that $2 \leq Q \leq 4$ ) representations of the algebra have special properties. One important one discussed below is the existence of a height representation, with positive and locally defined Boltzmann weights. Having $k$ integer is also desirable for the quantum loop models, because the existence of the Jones-Wenzl projector at these values means that the number of ground states remains finite even when the theory is defined on a torus [7].

In the loop representation of the Temperley-Lieb algebra, the generators act on $L$ strands, as shown in Fig. 1. The result is a completely packed set of self-avoiding and mutually-avoiding 


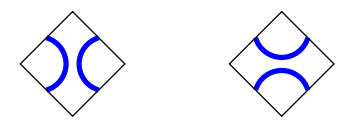

Figure 1: Action of $I$ (left picture) and $e_{i}$ (right picture) on a pair of strands at positions $i$ and $i+1$. The transfer (time) direction is upwards. Each picture corresponds to a plaquette of the dual lattice.

loops. Complete packing means that the loops cover every link of the square lattice; the only degrees of freedom are the two ways in which the loops avoid each other at each vertex of the lattice. The transfer matrix adds $L$ plaquettes to the dual lattice as illustrated in Fig. 2, so it is acting on a zig-zag row of $L$ plaquettes. Multiplying out the product in (2) makes it obvious that each configuration in the completely packed loop model appears with a coefficient which is just $(\sqrt{Q})^{\# \text { loops }}$ when $R_{i}=I+e_{i}$, and $(-\sqrt{Q})^{\# \text { loops }}$ when $R_{i}=I-e_{i}$. Note in particular that the parameter $k$ in (4) does not need to be an integer for the loop representation to be defined.
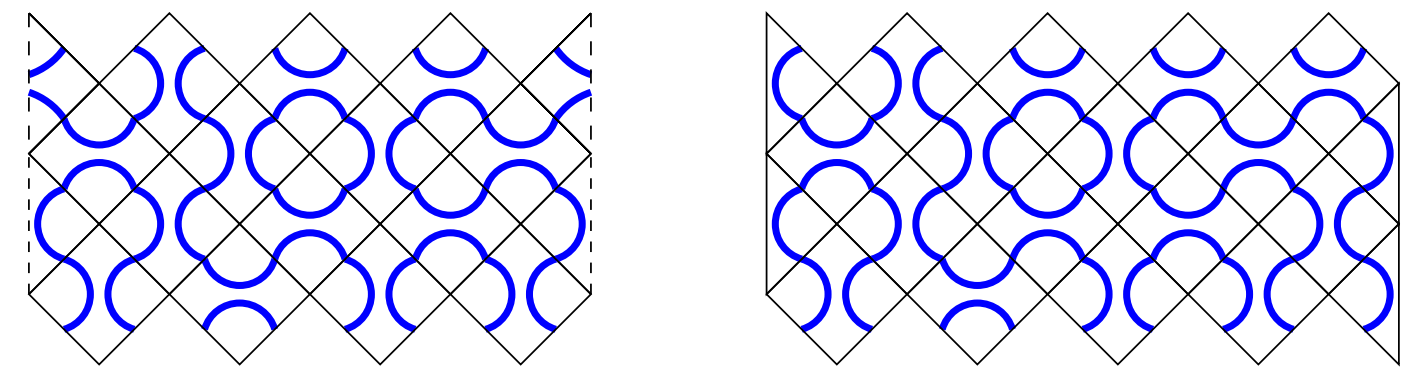

Figure 2: Action of a term in $T^{2}$ on a zig-zag row of $L$ plaquettes. The left (resp. right) picture illustrates periodic (resp. free, or reflecting) boundary conditions in the space direction.

A remarkable property of the loop representation is that the Boltzmann weight of a configuration depends only on topological properties of the loops. (In fact, for this reason the loop representation of the Temperley-Lieb algebra underlies the Jones polynomial in knot theory.) The local topological properties follow directly from the Temperley-Lieb algebra (11). The second relation means that configurations which are topologically equivalent receive the same weight, while he first relation means that a closed loop not wrapping around a cycle receives a weight $\sqrt{Q}$ relative to the configuration without the loop.

To evaluate the full partition function in the loop representation, we need to define the trace in (3), which corresponds to imposing periodic boundary conditions in the "time" direction. It is natural to require that closed loops wrapping around the time direction also receive a weight $\sqrt{Q}$. Thus the weight of a given loop configuration $\mathcal{L}$ is found simply by counting the number $n_{\mathcal{L}}$ of loops is the configuration:

$$
Z=\sum_{\mathcal{L}} Q^{n_{\mathcal{L}} / 2}
$$

This defines the Markov trace of elements of the Temperley-Lieb algebra. A loop is inherently a non-local object, so even though the Boltzmann weights here are all positive for $Q \geq 0$, the corresponding field theory is typically not unitary.

When $k$ is an integer, the Temperley-Lieb algebra has a height representation where the resulting lattice model (often known as RSOS, or restricted solid-on-solid, model) has positive 

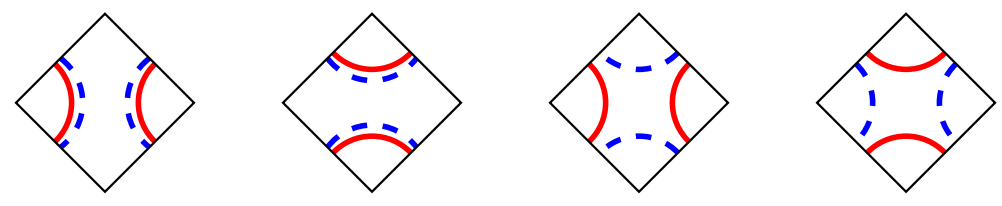

Figure 3: The four configurations at each vertex in the coupled completely packed loop model.

Boltzmann weights [13, 14, 15, 16]. The heights $h_{i}=1,2, \ldots, k+1$ live on the dual lattice, so that each plaquette illustrated in Fig. 2 has four heights at its corners. Heights of neighboring sites satisfy the RSOS constraint $\left|h_{i}-h_{j}\right|=1$. The generator $e_{i}$ now acts locally as

$$
e_{i}\left|h_{i-1}, h_{i}, h_{i+1}\right\rangle=\delta_{h_{i-1}, h_{i+1}} \sum_{h_{i}^{\prime}}\left(\frac{\sin \left(\frac{\pi h_{i}}{k+2}\right) \sin \left(\frac{\pi h_{i}^{\prime}}{k+2}\right)}{\sin \left(\frac{\pi h_{i-1}}{k+2}\right) \sin \left(\frac{\pi h_{i+1}}{k+2}\right)}\right)^{1 / 2}\left|h_{i-1}, h_{i}^{\prime}, h_{i+1}\right\rangle
$$

and the trace in (3) is simply the usual matrix trace.

The connection between the loop and height models - valid when both are defined, i.e., for $k$ integer - is based on exact partition function identities [15] that hold strictly speaking only when the underlying lattice is planar. Imposing doubly periodic boundary conditions leads to further subtleties, which are however not important in the context of this work.

This paper is devoted to studying two coupled self-dual Potts models, in both their loop and height formulations. We thus consider two Potts models on the same lattice, described respectively by two independent sets of Temperley-Lieb generators labeled by $e_{i}$ and $f_{i}$. Here we require that $Q$ be the same for the two models; later we will consider the more general case. We require that the coupled model remain self-dual under the combination of the duality transformations in both models, and that it be invariant under exchange of the two models. The transfer matrix remains of the form (2), with $R_{i}$ for each plaquette given by

$$
R_{i}=I \otimes I+e_{i} \otimes f_{i}+\lambda\left(I \otimes f_{i}+e_{i} \otimes I\right)
$$

In the loop representation, this is very simple to represent; the four possible configurations at each vertex are displayed in Fig. 3. The two copies mean that on each link there are two species of loops. Loops of each species cannot cross at vertices, but the two species can cross. The partition function is a sum over all configurations $\mathcal{L}$ and $\mathcal{M}$ of the two species of loops:

$$
Z=\sum_{\mathcal{L}} \sum_{\mathcal{M}} Q^{\left(n_{\mathcal{L}}+n_{\mathcal{M}}\right) / 2} \lambda^{n_{X}}
$$

where $n_{X}$ is the number of times the rightmost two vertices in Fig. 3 occur. This is a completely packed version of the loop model discussed in [17]; we will make contact with the results discussed there when we discuss the dilute generalization in section 5 ,

When the parameter $\lambda=1$ and $\lambda=-1$, we have decoupled ferromagnetic and antiferromagnetic models respectively, both critical. When $\lambda=0, R_{i}$ describes a single $Q^{2}$-state Potts model, because the products $e_{i} \otimes f_{i}$ generate a Temperley-Lieb algebra (11) with $Q$ replaced by $Q^{2}$. In the loop representation, $\lambda=0$ amounts to requiring that the loops in the two models be identical, so that each loop gets a weight $(\sqrt{Q})^{2}=Q$. Taking the dual of one of the two models exchanges $\lambda \mapsto 1 / \lambda$, so we restrict our attention to $|\lambda| \leq 1$. 
In the height representation, the theory remains unitary for $\lambda \geq-1 / \sqrt{Q}$. This follows by explicitly examining the Boltzmann weights; they all remain positive except for one type of plaquette. Namely, a negative Boltzmann weight occurs for $\lambda<0$ for all plaquettes where the heights in one RSOS model differ in the time direction, while the heights in the other RSOS model differ in the space direction. Because of the periodic boundary conditions in the time direction, there must be an even number of such plaquettes. Thus one can flip the sign of these weights without changing $Z$, giving a local model with all positive Boltzmann weights. This remains true until $\lambda=-1 / \sqrt{Q}$, where other weights change sign.

For $k=2$, perturbing $\lambda$ away from 1 is marginal and preserves the criticality. This is easy to see in the height formulation, because $k=2$ corresponds to $Q=2$ : (6) then describes two coupled Ising models. This is known as the Ashkin-Teller model along its self-dual line, and the physics is well understood in the height formulation [18]. The model remains critical until $\lambda=0$, where it is equivalent to the critical 4-state Potts model. For $\lambda<0$, it is no longer critical.

At the decoupled points for $Q \leq 4$, the models are critical and so described by a conformal field theory. In the height model for $\lambda=1$, the conformal field theories describing these critical models are known as the unitary minimal models, and have central charge [14]

$$
c=2\left(1-\frac{6}{(k+1)(k+2)}\right) \quad \text { for } \lambda=1
$$

The behavior of the decoupled height models at the antiferromagnetic critical point $\lambda=-1$ is somewhat trickier to describe. The negative Boltzmann weights make the theory non-unitary. However, if one instead uses a Hamiltonian formulation of a $1+1$ dimensional quantum theory, the Hamiltonian corresponding to $\lambda=-1$ is simply $H=\sum_{i=1}^{L} e_{i}$, which is hermitian. The unitary conformal field theory describing the continuum limit of this Hamiltonian is known as the $Z_{k}$ parafermion model, whose central charge is

$$
c_{\text {height }}=2\left(\frac{2(k-1)}{k+2}\right) \quad \text { for } \lambda=-1 \text {. }
$$

In the loop model, (8) remains true for $\lambda=1$. However, since $k$ is now a continuous parameter, the cases $\lambda=1$ and $\lambda=-1$ are now linked by analytic continuation, $k \mapsto-\frac{k}{k+1}$. Note that under this transformation $\sqrt{Q} \mapsto-\sqrt{Q}$, which is tantamount to $R_{i}=I+e_{i} \mapsto I-e_{i}$ as required. In particular, for $\lambda=-1$ the correct central charge is then obtained by analytically continuing (8) and reads

$$
c_{\text {loop }}=2\left(1-\frac{6(k+1)^{2}}{k+2}\right) \quad \text { for } \lambda=-1 .
$$

When $k$ is integer, the corresponding critical theory completely disappears from $Z$ due to massive cancellations [19] of eigenvalues in the Markov trace (3), and what remains is tantamount to the height model.

Taking $\lambda$ away from 1 is a perturbation odd under each the individual dualities of the Potts models, and even under the combination. The simplest operator with these properties is given by the product of the energy operators $\varepsilon_{1} \varepsilon_{2}$ of the two models. This operator has dimension $(k+4) /(k+1)$, so it is relevant for $k>2$; it is the only relevant one with the appropriate symmetries. Thus the question becomes whether this perturbation causes a flow to a non-trivial critical point. Compelling evidence suggests that there is no such critical point, although no definitive argument rules it out, Namely, this perturbation is integrable in the field theory [21], and the only consistent $S$ matrices describing this perturbed theory are massive, indicating there 


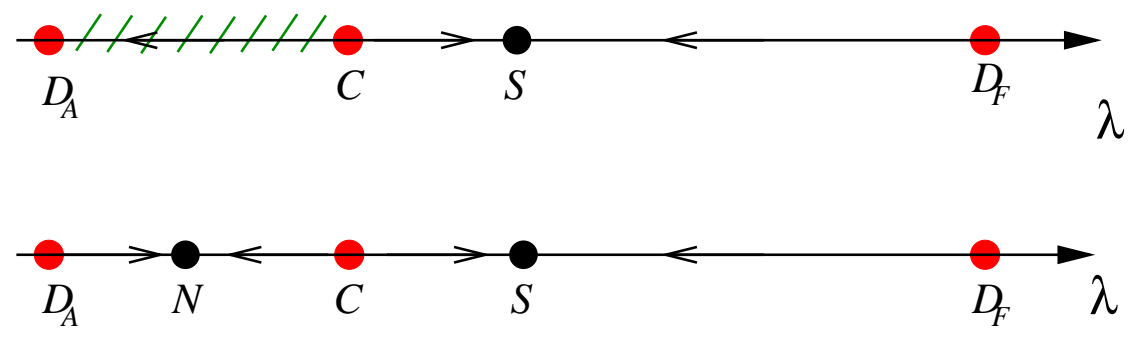

Figure 4: Phase diagram for the loop model (top) and the height model (bottom). The arrows indicate renormalization group flows. The cross-hatching between the new critical point $C$ and the decoupled antiferromagnetic critical point $D_{A}$ represents the critical phase.

is no flow to a critical point. 1 Moreover, this is consistent with the fact that at $\lambda=0$, the model for $k>2$ is non-critical, because the $Q^{2}$-state Potts model at its self-dual point is critical only for $Q \leq 2$. Thus it is natural to guess that for $k>2$, the flow goes from $\lambda=1$ to $\lambda=0$, the latter being a stable non-critical fixed point of the renormalization group. Our numerical work supports this picture.

The interesting new physics occurs for $\lambda$ negative, where we find a new critical point. We present this calculation in section 3, where we derive the exact location of the critical point and determine which conformal field theory describes its continuum limit.

The arguments given above - in conjunction with numerical calculations presented in section 3.2 below - lead to the phase diagrams shown in Fig. 4. The point $S$ is the strongly coupled point $\lambda=0$, which is non-critical for $k>2$. The point $C$ is the new critical point at $\lambda_{c}$, to be discussed in detail in section 3 below. The points $D_{F}$ and $D_{A}$ are the decoupled ferromagnetic and antiferromagnetic critical points at $\lambda=1$ and $\lambda=-1$. The characteristics of $D_{A}$ differ between the height and the loop models. Finally, the point $N$ is a non-critical point which occurs only in the height model, and which is required for the consistency of its RG flow diagram.

Note in particular that the phase $\lambda \in\left[-1, \lambda_{c}\right)$ is non-critical in the height model, but critical in the loop model. The latter is an example of a "Berker-Kadanoff" phase [19].

The point $\lambda=-1 / \sqrt{Q}$, the lowest value of $\lambda$ where the height representation is unitary, is special. It is quite important for quantum loop models, and it lies in the critical phase for $k<6$, and is the new critical point for $k=6$. When $\lambda=-1 / \sqrt{Q}$, the weight of each configuration can explicitly be given in terms of a chromatic polynomial squared [6]. This can be seen for the square lattice by noting that

$$
\begin{aligned}
R & =(1-e / d)(1-f / d)+\left(d^{2}-1\right)(e / d)(f / d) \\
& =(e-1 / d)(f-1 / d)+\left(d^{2}-1\right)\left(1 / d^{2}\right)
\end{aligned}
$$

where $d=\sqrt{Q}$. The projectors $1-e / d$ and $e / d$ are orthogonal. We divide the square lattice on which the loops live into the usual two sublattices (even and odd). The low-temperature expansion of a single Potts model is found from writing $R_{\text {Potts }}=(1-e / d)+(d-1) e / d$ on sublattice 1 , and $R_{\text {Potts }}=(e-1 / d)+(d-1) / d$ on sublattice 2 , and then associating a domain wall with each $1-e / d$ term included on sublattice 1 , and $e-1 / d$ on sublattice 2 . It is easy to check that these walls cannot end, although there can be three or four walls touching each

\footnotetext{
${ }^{1}$ We note, however, that the $S$ matrices given in [21] for $k>2$ are wrong - they are the level-rank duals of the correct ones, in the technical sense described below.
} 
site. If we sum over the spins for any fixed domain-wall configuration, the weight per graph is proportional to the chromatic polynomial of the dual lattice. This is easiest to see by simply proving it for all $Q$ integer in the original Potts representation; by definition of the chromatic polynomial it must apply for all $Q$. Thus coupling Potts models at $\lambda=-1 / d$ corresponds to requiring that the low-temperature expansion of the two be the same. (This is proved for any lattice with four links touching each site in [6].) The behavior of the low-temperature expansion at $\lambda=-1 / d$ is therefore analogous to that of the completely packed (Fortuin-Kasteleyn [20]) loop expansion at $\lambda=0$.

\section{The new self-dual critical point}

In this section we show that at a particular value $\lambda=\lambda_{c}$, the coupled Potts models have a critical point. In accord with the discussion of the previous section, this value must be negative, and we show here that

$$
\lambda_{c}=-\sqrt{2} \sin \left(\frac{\pi(k-2)}{4(k+2)}\right) .
$$

The conformal field theory describing this critical theory is the coset model 22 ]

$$
\frac{S U(2)_{k} \times S U(2)_{k}}{S U(2)_{2 k}}
$$

with central charge

$$
c=\frac{3 k^{2}}{(k+1)(k+2)} .
$$

\subsection{Level-rank duality and the BMW algebra}

At the value $\lambda=\lambda_{c}$, the coupled theory is integrable. We show this by using level-rank duality to write the transfer matrix here in terms of another algebra, the $S O(N)$ Birman-Murakami-Wenzl (BMW) algebra [23]. Lattice models involving this algebra were introduced long ago [24], and it is possible to show that integrability follows directly from algebraic properties, and so applies to any representation [25. In this more general setting, the Andrews-Baxter-Forrester height models [13. discussed are associated with $S U(2)$ and its quantum-group algebra deformation $U_{q}(S U(2))$.

The BMW algebra was introduced to study representations of the braid group and find new knot and link invariants. Braid generators in general satisfy $B_{i} B_{j}=B_{j} B_{i}$ for $|i-j|>1$, and

$$
B_{i} B_{i+1} B_{i}=B_{i+1} B_{i} B_{i+1} .
$$

This is known as the "third Reidemeister move" in knot theory. The braid generators can be inverted: $B_{i} B_{i}^{-1}=I$ (the second Reidemeister move). A useful graphical representation of the braid group is given by each $B_{i}$ and $B_{i}^{-1}$ as acting on two strands like the Temperley-Lieb generators, but where $B$ and $B^{-1}$ correspond to overcrossings and undercrossings respectively.

The $S O(N)$ BMW algebra is generated by the $B_{i}$ and $B_{i}^{-1}$, where $i=1,2, \ldots, L$. This algebra involves a parameter $q$ as well as $N$; for the models we label $S O(N)_{k}, q=e^{i \pi /(N+k-2)}$ is a root of unity. The braid generators of the BMW algebra of course satisfy the second and third Reidemeister moves, but the algebra has more structure. The Temperley-Lieb algebra is a subalgebra, with $E_{i}$ defined as

$$
E_{i}=I+\frac{B_{i}-B_{i}^{-1}}{q-q^{-1}}
$$


where $q$ is a parameter. Here the $E_{i}$ obey

$$
\left(E_{i}\right)^{2}=D E_{i}, \quad E_{i} E_{i+1} E_{i}=E_{i}, \quad E_{i} E_{j}=E_{j} E_{i} \text { for }|i-j|>1
$$

where

$$
D=1+\frac{q^{N-1}-q^{1-N}}{q-q^{-1}} .
$$

The full set of BMW relations can be found in [25]. One useful one is the first Reidemeister move

$$
B_{i} E_{i}=E_{i} B_{i}=q^{N-1} E_{i} .
$$

For later use, it is convenient to define

$$
X_{i}=q^{-1} I+q E_{i}-B_{i}
$$

This generator $X_{i}$ is particularly interesting in the $N=3$ case, where the BMW algebra is related to the chromatic algebra [26, 27].

The coupled Potts model transfer matrix (6) can be written in terms of the $S O(4)_{k}$ BMW algebra. This BMW algebra is special because the algebra $S O(4)$ is the direct product of two $S U(2)$ algebras. One should think of the strands in $S O(N)_{k}$ as behaving in the vector representation of the quantum-group algebra $U_{q}(S O(N))$. As with ordinary Lie algebras, $U_{q}(S O(4))$ decomposes into copies of $U_{q}(S U(2))$, so the lines in $S O(4)_{k}$ behave as two independent spin$1 / 2$ lines in $S U(2)_{k}$. Spin-1/2 lines obeying the Temperley-Lieb algebra satisfy the braid group relation $b_{i} b_{i+1} b_{i}=b_{i+1} b_{i} b_{i+1}$ and $b_{i} b_{i}^{-1}=I$ if we define the $b_{i}$ via the "skein relation"

$$
b_{i}=q^{-1 / 2}-q^{1 / 2} e_{i}, \quad b_{i}^{-1}=q^{1 / 2}-q^{-1 / 2} e_{i},
$$

where the $e_{i}$ obey (11) for $\sqrt{Q}=q+q^{-1}$. (This is the braid-group representation underlying the Jones polynomial.) So less abstractly, given two independent representations labeled $e_{i}$ and $f_{i}$ of the TL algebra, we can construct one of the $S O(4)_{k}$ BMW algebra by defining

$$
\begin{aligned}
E_{i}^{(4)} & =e_{i} \otimes f_{i} \\
B_{i}^{(4)} & =\left(q^{-1 / 2} I-q^{1 / 2} e_{i}\right) \otimes\left(q^{-1 / 2} I-q^{1 / 2} f_{i}\right)=q^{-1} I+q E_{i}^{(4)}-X_{i}^{(4)}
\end{aligned}
$$

where given the definition of $X_{i}$ in (16), we have

$$
X_{i}^{(4)}=e_{i} \otimes I+I \otimes f_{i}
$$

for this representation of $S O(4)_{k}$. Therefore the transfer matrix (6) for each plaquette can be written simply as

$$
R_{i}=I+E_{i}^{(4)}+\lambda X_{i}^{(4)} .
$$

Because of the relation (15), we need use only three of the generators for each plaquette.

Lattice models whose transfer matrices are built on the $S O(N)_{k}$ BMW algebra are integrable only for special values of the parameters. Height models associated with all non-exceptional Lie algebras were introduced in [24]. The transfer matrices for the critical points of these lattice models can be written in terms of BMW algebras; for the $S O(N)_{k}$ case they are [25]

$$
R_{i}^{(N)}(u)=[\gamma-u][1-u] I+[u][1-\gamma+u] E_{i}+[\gamma-u][u] X_{i}
$$


where $\gamma=(N-2) / 2$ and we have introduced the shorthand

$$
[x]=\sin \left(\frac{\pi x}{N+k-2}\right)=\frac{1}{2}\left(q^{x}-q^{-x}\right) .
$$

The parameter $u$ is simply a lattice anisotropy which does not affect any of the universal properties. Under 90 degree rotations, $I$ and $E$ are interchanged, while $X$ remains invariant. Thus isotropic Boltzmann weights are found by setting $u=\gamma / 2$, giving

$$
\frac{R_{i}^{(N)}(\gamma / 2)}{[\gamma / 2][1-\gamma / 2]}=I+E_{i}+\frac{[\gamma / 2]}{[1-\gamma / 2]} X_{i}
$$

From the BMW algebra it follows that this $R$ matrix satisfies the Yang-Baxter equation

$$
R_{i}(u) R_{i+1}\left(u+u^{\prime}\right) R_{i}\left(u^{\prime}\right)=R_{i+1}\left(u^{\prime}\right) R_{i}\left(u+u^{\prime}\right) R_{i+1}(u),
$$

so that the model is integrable. Note that the braid generators are recovered in the $u \rightarrow \pm \infty$ limits:

$$
\lim _{u \rightarrow \pm \infty} \frac{R_{i}^{(N)}(u)}{q^{ \pm(u-\gamma)}}=q^{\mp 1} I+q^{ \pm 1} E_{i}-X_{i}=B_{i}^{ \pm 1} .
$$

It is straightforward to establish that lattice models with the transfer matrix given by (20) and (2) are critical. This is a generic feature of integrable models with trigonometric Boltzmann weights. Here one can see this by utilizing a deformation of these models which preserves the integrability [24]. This enables one for example to compute the free energy by using the inversionrelation method [18]. From this one sees that as the deformation parameter is tuned back to the trigonometric weights, the free energy behaves non-analytically, as it does at a critical point. The central charge of the conformal field theory describing this critical point can be computed after making some (very believable) technical assumptions; for $u>0$ it is [28]

$$
c=\frac{N}{2}\left(1-\frac{N^{2}-3 N+2}{(N+k-2)(N+k-3)}\right)
$$

which is the central charge of the

$$
\frac{S O(N)_{k-1} \times S O(N)_{1}}{S O(N)_{k}}
$$

coset models.

The new critical point for coupled Potts models does not correspond to the $N=4$ case of (21). Rather, this yields the decoupled ferromagnetic critical point. Namely, when $N=4, \gamma=1$, and the decomposition of the $S O(4)$ BMW algebra in terms of two Temperley-Lieb algebras in (17/18) means

$$
R_{i}^{(4)}(u)=\left([1-u] I+[u] e_{i}\right) \otimes\left([1-u] I+[u] f_{i}\right)
$$

Thus the models decouple here: at the isotropic point $u>0$ this is simply the $\lambda=1$ ferromagnetic critical point. Indeed, because as conformal field theories $S O(4)_{k}=S U(2)_{k} \times S U(2)_{k}$, the coset in (22) simply splits into two copies of

$$
\frac{S U(2)_{k-1} \times S U(2)_{1}}{S U(2)_{k}}
$$


with central charge (8) .

To find the new critical point at $\lambda=\lambda_{c}$, we need to exploit the level-rank duality of the BMW algebra [27]. It is simple to check that if the $B_{i}$ satisfy this $S O(N)_{k}$ algebra, then the $-B_{i}^{-1}$ satisfy the $S O(k)_{N}$ algebra. This follows because $q$ is invariant under this exchange, and

$$
q^{N-1} q^{k-1}=-1
$$

so that $D$ remains unchanged. Thus from any representation of $S O(N)_{k}$ with generators $\left\{B_{i}, E_{i}\right\}$, we can construct one of $S O(k)_{N}$ with generators $\left\{\widetilde{B}_{i}, \widetilde{E}_{i}\right\}$ by

$$
\widetilde{B}_{i}=-B_{i}^{-1}, \quad \widetilde{E}_{i}=E_{i} .
$$

We then have

$$
\begin{aligned}
\widetilde{X}_{i} & =q^{-1} I+q E_{i}+B_{i}^{-1} \\
& =q^{-1} I+q E_{i}+\left(q-q^{-1}\right)\left(I-E_{i}\right)+B_{i} \\
& =\left(q+q^{-1}\right)\left(I+E_{i}\right)-X_{i}
\end{aligned}
$$

The transfer matrix of the coupled Potts models defined in (6) has been expressed in terms of the $S O(4)_{k}$ BMW algebra via (19). The level-rank duality therefore implies that it can also be written in terms of the generators $\left\{\widetilde{E}_{i}, \widetilde{X}_{i}\right\}$ of the $S O(k)_{4}$ BMW algebra:

$$
R_{i}=\left(1+\lambda\left(q+q^{-1}\right)\right)\left(I+\widetilde{E}_{i}\right)-\lambda \widetilde{X}_{i}
$$

We can now find the new critical point, because the $S O(k)_{4}$ models are critical when the Boltzmann weights obey (21). It follows that the coupled Potts models are critical at $\lambda=\lambda_{c}$, where $R_{i} \propto R_{i}^{(k)}$. This holds if

$$
-\frac{\lambda_{c}}{1+\lambda_{c}\left(q+q^{-1}\right)}=\frac{[\gamma / 2]}{[1-\gamma / 2]}
$$

Plugging in the appropriate values of $[\gamma / 2]$ and $[1-\gamma / 2]$ for $S O(k)_{4}$ and solving for $\lambda_{c}$ gives (11).

We expect the central charge to be independent of the representation of the BMW algebra used, as long as appropriate boundary conditions are chosen. This critical point at $\lambda=\lambda_{c}$ therefore should have the central charge of the coset conformal field theory

$$
\frac{S O(k)_{3} \times S O(k)_{1}}{S O(k)_{4}}
$$

which is that given in (13). A related type of level-rank duality arises in coset conformal field theories 29]. This lets us map this coset model on to others:

$$
\frac{S O(k)_{3} \times S O(k)_{1}}{S O(k)_{4}} \approx \frac{S O(4)_{k}}{S O(3)_{k}}=\frac{S U(2)_{k} \times S U(2)_{k}}{S U(2)_{2 k}} .
$$

The latter coset is the one we gave above in (12); note that the coset description involving $S O(4)$ is not the $S O(4)$ case of (22). Thus exploiting the integrability of this lattice model lets us not only determine the location of the critical point exactly, but it lets us determine the correct coset conformal field theory.

Note that when $N=k$, the level-rank duality does not change the model, only the couplings. The points $\lambda=1$ and $\lambda=\lambda_{c}$ are not self-dual under level-rank duality, but rather are mapped to 


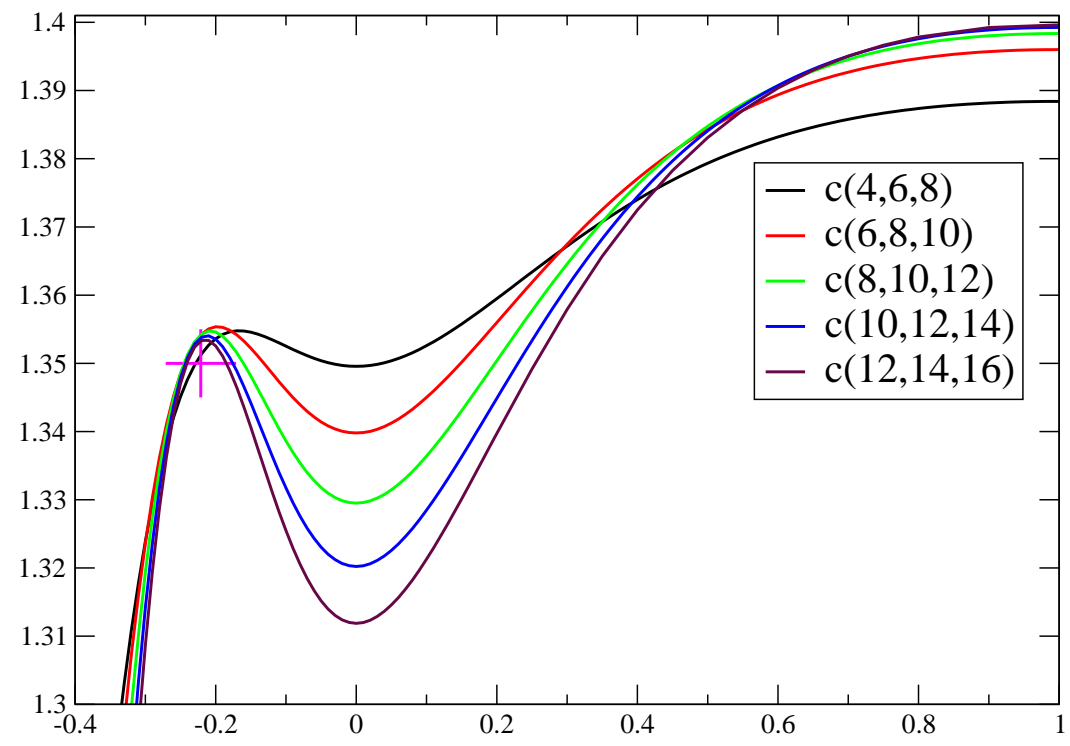

Figure 5: Central charge of the loop model with $k=3$, as a function of $\lambda$. The pink cross indicates the expected infinite-size behavior at the new self-dual critical point.

each other. Thus for the coupled Potts models with $N=k=4(Q=3)$, the new critical point at $\lambda=\lambda_{c}$ is equivalent to the usual $\lambda=1$ pair of decoupled models. Indeed, the central charge (13) here at $\lambda=\lambda_{c}$ is $8 / 5$, that of two decoupled 3-state Potts models at their ferromagnetic critical points. As pointed out in section 5.2.2 of [9], the coupled $Q=3$ models in fact have an exact symmetry $J \mapsto-J$, where $J$ is the original Potts spin coupling. In the present notation we have $\lambda=\left(\mathrm{e}^{J}-1\right) / \sqrt{Q}$, and the symmetry reads $\lambda \mapsto-\frac{\lambda}{1+\lambda \sqrt{Q}}$. For $Q=3$ this gives indeed $1 \mapsto-\frac{1}{1+\sqrt{3}}=\lambda_{c}$.

\subsection{Numerical calculations}

The coupled model can be studied numerically using techniques for exactly diagonalizing the transfer matrix $T$, either in the loop or the height representation. We focus here on the loop model.

For reasons of numerical efficiency, it turns out to be most convenient to chose the transfer direction to be axial with respect to the square lattice on which the loops live (as opposed to the diagonal transfer direction depicted in Figs. 2 (3). Periodic boundary conditions are imposed in the space direction. To avoid parity effects in the antiferromagnetic region, the number of strands $L$ is chosen to be even. We can then handle widths up to $L=18$.

The central charge can be extracted from finite-size scaling of the leading eigenvalue of $T$ in the standard way. The resulting three-point fits $c(L-4, L-2, L)$ are shown in Fig. [5 as functions of $\lambda$. The figure shows the case $k=3$, but larger integer values of $k$ lead to similar 


\begin{tabular}{l|lll} 
Exponent & $k=3$ & $k=4$ & $k=5$ \\
\hline$x_{2,0}$ & $0.998(1)$ & $0.999(1)$ & $0.999(1)$ \\
$x_{2,2}$ & $0.200(2)$ & $0.130(2)$ & $0.095(2)$ \\
$x_{4,0}$ & $1.560(2)$ & $2.5(2)$ & - \\
$x_{4,2}$ & $0.774(1)$ & $0.510(5)$ & $0.392(4)$ \\
$x_{4,4}$ & $0.926(4)$ & $0.677(2)$ & $0.555(5)$ \\
$x_{6,0}$ & $2.40(3)$ & $2.47(1)$ & $3.0(1)$ \\
$x_{6,2}$ & $1.70(5)$ & $1.15(5)$ & $0.84(5)$ \\
$x_{6,4}$ & $1.85(5)$ & $1.28(5)$ & $1.00(5)$ \\
$x_{6,6}$ & $2.08(7)$ & $1.54(5)$ & $1.26(6)$
\end{tabular}

Table 1: Watermelon exponents $x_{\ell_{1}, \ell_{2}}$ at the critical point $\lambda_{c}$ for $k=3,4,5$. The error bar on the last quoted digit is given in parentheses. One entry is missing due to convergence problems in the diagonalization method being used.

results. Eq. (8) then predicts $c=\frac{7}{5}=1.4$ at the decoupled ferromagnetic point $\lambda=1$, and Eq. (13) gives $c=\frac{27}{20}=1.35$ at the new self-dual critical point, which according to (11) is situated at $\lambda_{c}=-\sqrt{2} \sin \left(\frac{\pi}{20}\right) \simeq-0.2212$. Finally, for the strongly coupled point $\lambda=0$ we have a single $Q^{2}=\left(2 \cos \left(\frac{\pi}{5}\right)\right)^{4} \simeq 6.8541$ state Potts model, which is non-critical but with a rather large correlation length.

All of this is nicely brought out by Fig. 5. The fact that the peaks in $c$ at the critical points $\lambda=\lambda_{c}$ and $\lambda=1$ narrow upon increasing $L$, further indicates that both points are RG repulsive.

To summarize this far, our numerical results not only confirm the existence of the critical point at $\lambda=\lambda_{c}$, but also provide compelling evidence that the central charge (13) is the correct one.

We now turn to the operator content of the critical theory. One of the most interesting and geometrically appealing operators that can be defined in a loop model is the so-called $\ell$-leg watermelon operator $\mathcal{O}_{\ell}$ [30]. To define this operator properly, we first imagine assigning an arbitrary orientation (clockwise or counterclockwise) to each loop in the model; the original loop model is then recovered by independently summing over the two orientations of each loop. The oriented loop model satisfies a zero-divergence constraint: the net arrow flow out of any simply connected region vanishes. The operator $\mathcal{O}_{\ell}$ breaks this constraint, and corresponds to a point (or a small neighborhood of a point) that acts as a source of $\ell$ oriented loop segments. The critical exponents $x_{\ell}$ corresponding to the asymptotic decay of the two-point correlation functions

$$
\left\langle\mathcal{O}_{\ell}\left(\mathbf{x}_{1}\right) \mathcal{O}_{-\ell}\left(\mathbf{x}_{2}\right)\right\rangle \sim\left|\mathbf{x}_{1}-\mathbf{x}_{2}\right|^{-2 x_{\ell}} \quad \text { for }\left|\mathbf{x}_{1}-\mathbf{x}_{2}\right| \ll 1
$$

can be computed analytically when the loop model admits a Coulomb gas construction. This has been accomplished for the loop model corresponding to a single Potts model [30] as well as for more complicated loop models [10].

The context of $\mathcal{N}$ coupled loop models allows for generalizing these operators to $\left(\ell_{1}, \ell_{2}, \ldots, \ell_{\mathcal{N}}\right)$ leg watermelon operators $\mathcal{O}_{\ell_{1}, \ell_{2}, \ldots, \ell_{\mathcal{N}}}(\mathbf{x})$, where the $p$ th model supports an $\ell_{p}$-leg defect at the point $\mathbf{x}$. The interpretation of this generalization in the replica setup was given in [9]. The corresponding critical exponents can be measured numerically by defining a modified transfer matrix in which the $p$ th model is constrained to having precisely $\left|\ell_{p}\right|$ loops which wind around the time direction. 
Returning to the case $\mathcal{N}=2$, we have determined numerically the exponents $x_{\ell_{1}, \ell_{2}}$ (with $\ell_{p}=0,2,4,6$ for $p=1,2$ ) at the critical point (11). This study involved strips of width up to $L=16$ strands. Results for parameter values $k=3,4,5$ are shown in Table 1 , These results give very strong support for the following conjectures

$$
x_{2,0}=1, \quad x_{2,2}=\frac{4}{(k+1)(k+2)}
$$

and moderately strong support for the additional conjecture

$$
x_{4,2}=\frac{16}{(k+1)(k+2)} .
$$

The denominator $(k+1)(k+2)$ of these expressions is in accord with heuristic arguments; operators arising in the unitary coset conformal field theories (12) describing these critical points indeed have dimensions with this denominator.

\section{The critical phase}

The phase diagram for $\lambda<\lambda_{c}$ is different for the height and loop models. To understand this, let us first study the region around $\lambda=-1$, perturbing away from the decoupled antiferromagnetic self-dual critical point. The situation is much subtler than at $\lambda=1$, because the models for $\lambda<-1 / d$ are not unitary. We argue here that the perturbation around $\lambda=-1$ is relevant for the height model, but irrelevant for the loop model.

Recall that the operator $\varepsilon_{1} \varepsilon_{2}$ responsible for the flow away from $\lambda=1$ has dimension $(k+$ $4) /(k+1)$, and so is relevant for $k>2$. In the loop model, the points $\lambda=1$ and $\lambda=-1$ are linked by the analytic continuation $k \mapsto-\frac{k}{k+1}$, and so $\varepsilon_{1} \varepsilon_{2}$ has dimension $3 k+4$ and is irrelevant for any $k \geq 0$. The decoupled point $\lambda=-1$ should therefore act as an RG attractor for a whole range of parameter values $\lambda \in\left[-1, \lambda_{0}\right)$, i.e., we have a critical phase with constant values of the critical exponents.

The existence of a critical phase in the loop model is verified numerically in Fig. 6 for the case $k=3$. The central charge then reads, according to (10), $c=-\frac{182}{5}=-36.4$, in fine agreement with the numerics despite of the large corrections-to-scaling effects.

We next examine more closely the extent of the critical phase, and the nature of the transition at $\lambda_{0}$. Fig. 7 shows (still for $k=3$ ) the free energy per unit area $f(L)=-\frac{1}{L} \log \lambda_{\max }$ for various strip widths $L$, where $\lambda_{\max }$ is the dominant eigenvalue of the corresponding transfer matrix. For $L \geq 8$ there is a jump singularity in the derivative $f^{\prime}(\lambda)$ at some $\lambda=\lambda_{0}(L)$, signaling a first-order phase transition. It is this singularity which marks the termination of the critical phase.

The existence of this first-order phase transition can be argued analytically as well [31].

Indeed, at $\lambda=-1$ the $k \mapsto \tilde{k} \equiv-\frac{k}{k+1}$ transformation implies that the dominant eigenvalue of the transfer matrix corresponds to a sector of non-trivial topological charge. Specifically, the smallest (i.e., most negative) critical exponent corresponds, for $0<\tilde{k}<2$, to a single Potts cluster wrapping around both the space and time directions of the lattice (and thus there are zero wrapping cluster boundaries), while for $\tilde{k}>2$ it corresponds to two Potts clusters (hence four cluster boundaries) wrapping around the time direction. Needless to say, in the probabilistic regime $(\lambda>0)$ the dominant eigenvalue corresponds to the identity operator, and so no cluster wraps around the time direction (for finite $L$, and in the limit $M \rightarrow \infty$ ). We conclude that for some $\lambda_{0}(L) \in(-1,0)$ there must be a level crossing in the transfer matrix spectrum, corresponding to a first-order transition. 


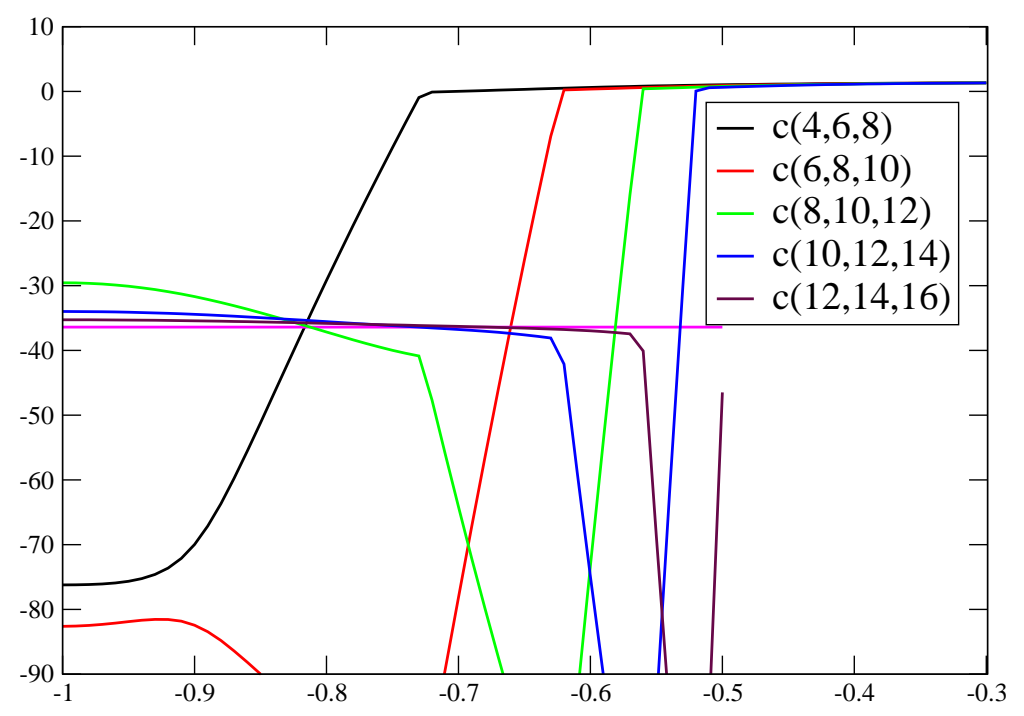

Figure 6: Central charge of the loop model with $k=3$, as a function of $\lambda$. The pink horizontal line indicates the expected infinite-size behavior throughout the critical phase.

For $k=3$ we have numerically determined

$$
\lambda_{0}(L)=-0.72793,-0.62225,-0.56084,-0.51985,-0.49002 \quad \text { for } L=8,10,12,14,16 .
$$

In the $L \rightarrow \infty$ limit this extrapolates to $\lambda=-0.25 \pm 0.05$, in conspicuous agreement with $\lambda_{c} \simeq-0.2212$ from (11). Similar agreements are found for other values of $k$.

We are therefore led to conjecture that $\lambda_{0}=\lambda_{c}$ for any $k$. This means that at the critical point $\lambda_{c}$ the loop model stands at a continuous first-order phase transition [32], while the height model stands at a conventional second-order transition. The two transitions are in the same universality class. This scenario is closely analogous to the one found for the antiferromagnetic transition in a single Potts model [33, 34].

Turning now to the height model, a perturbation away from $\lambda=-1$ in the $Z_{k}$ parafermion theory (9). As with the perturbation around $\lambda=-1$, the perturbing operator here must be odd under the individual dualities of the models, and even under the combination. In the unitary Hamiltonian formulation, this corresponds to the product of energy operators in the parafermion theories, which is relevant with dimension $4 /(k+2)$. Accordingly, we expect a different scenario in which the critical phase does not exist. Numerical transfer matrix diagonalization reveals that the free energies $f(L)$ are smooth functions all the way down to $\lambda=-1$. For $\lambda>\lambda_{0}(L)$ they are identically equal to those of the loop model. Exactly at $\lambda=-1$ there is a jump singularity in the derivative $f^{\prime}(\lambda)$, as is consistent with the overall $\lambda \mapsto 1 / \lambda$ symmetry of the model. [At $\lambda=1$ the Perron-Frobenius theorem prevents such a singularity from occurring.] In between $\lambda=-1$ and $\lambda=\lambda_{c}$ the behavior is non-critical, the effective central charge being zero. 


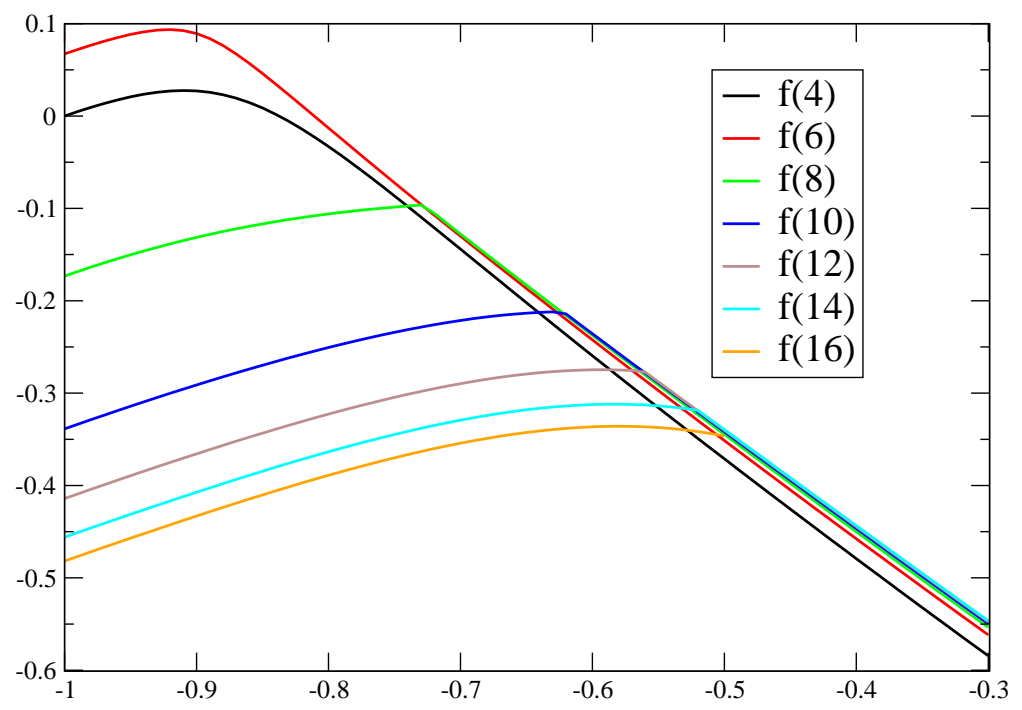

Figure 7: Free energies $f(L)$ of the loop model with $k=3$, as functions of $\lambda$. Level crossings are visible for strip widths $L \geq 8$.

\section{Dilute loops}

The loop models we have studied thus far are completely packed: every site of the lattice is covered by strands. It is natural to relax this constraint, and consider "dilute" loops. We show in this section that the critical point and phase still persist for fairly large amounts of dilution.
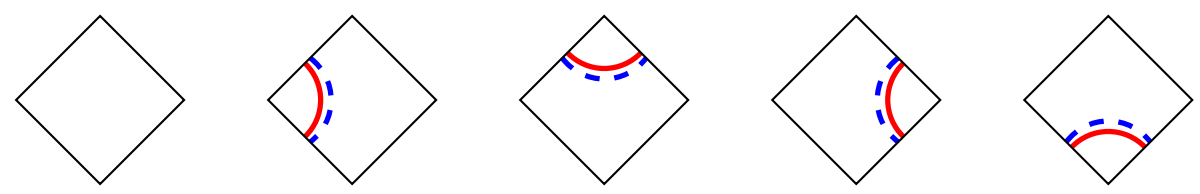

Figure 8: The five additional configurations at each vertex in the dilute loop model.

The dilute model we discuss here requires that loops of the two species be on the same links: they are "glued" together. This does not reduce to a single loop model, because when two loops meet at the same vertex, the four possibilities in Fig. 3 are still possible. For reasons we will discuss below, the physics does not seem to be changed by whether or not one allows vertices with the pair of loops going straight through. Thus for simplicity, we still require that loops turn by 90 degrees at each vertex, so that there are nine allowed configurations at each vertex. The five new possibilities are displayed in Fig. 8, in addition to the empty vertex, there are four vertices where two links touching each vertex are empty. 
Let the weight of the vertices in Fig. 8 (from left to right) be

$$
\omega_{1}=1 \quad \text { and } \quad \omega_{2}=\omega_{3}=\omega_{4}=\omega_{5}=K
$$

and those of the vertices in Fig. 3 (from left to right) be

$$
\omega_{6}=\omega_{7}=K^{2} \beta \quad \text { and } \quad \omega_{8}=\omega_{9}=K^{2} \beta \lambda .
$$

Then $K$ roles as the fugacity per monomer, $\beta$ is a contact interaction, and $\lambda$ occurs when different loop species cross as before. We expect the contact interaction to be irrelevant, and so we set $\beta=1$ in what follows. The dilute coupled loop models therefore have partition function

$$
Z_{\text {dilute }}=\sum_{\mathcal{L}} \sum_{\mathcal{M}} K^{N_{\mathrm{m}}} Q^{\left(n_{\mathcal{L}}+n_{\mathcal{M}}\right) / 2} \lambda^{n_{X}}
$$

where $N_{\mathrm{m}}$ is the total number of monomers, and the sum is over dilute loops with the vertices described above. The completely packed model is recovered in the limit $K \rightarrow \infty$.

A critical point in this dilute loop model was discussed at length in [17]. There several indirect arguments were given as to why this dilute loop model will have a critical point described precisely by the same coset conformal field theory (12) as we showed above described the critical point at $\lambda=\lambda_{c}$ in the completely packed limit. In this section we review these arguments, use numerics to provide further strong support for them, and map out the full phase diagram of the dilute loop model.

The arguments presented in [17] were the reverse of those presented above. In [17], critical theories known to describe the coset models (12) were shown to be closely related to this dilute loop model. One argument involves an integrable lattice height model [35, 36] which has a critical point whose continuum limit is described by the coset (12). It was shown that in a certain offcritical limit, the height model reduces precisely to the dilute loop model. An integrable line of couplings connects this limit to the critical point, so that the corner-transfer matrix eigenvalues and hence the local height probabilities can be computed along this line. The configurations which dominate this computation are precisely those of the dilute loop model. Moreover, the remaining configurations can be described by a more general dilute loop model, where multiple strands of each species are allowed on a link. The fact that the configurations with multiply occupied links do not dominate the corner-transfer matrix computation is a strong sign that at the critical point, allowing or disallowing such configurations is irrelevant. Thus the dilute loop model discussed above should still have a critical point described by (12).

Another argument briefly mentioned in [17] comes from studying the $S$ matrix of the integrable field theory at and near this critical point. A compelling picture of the relation between loop models and integrable $S$ matrices was presented in [37]. There it was argued that one could think of the worldlines of particles in the integrable $1+1 d S$ matrix description as dilute loops in the corresponding classical $2 \mathrm{~d}$ lattice model. This observation was made precise in [38], where it was shown that the $S$ matrices for the $\Phi_{1,3}$ perturbation of the minimal models can be expressed in terms of the Temperley-Lieb generators, which in turn have the loop representation described in section (2). For the $S$ matrix $\mathcal{S}_{p}$ for the minimal model of central charge $c=1-6 /[(p+$ $1)(p+2)]$, the weight of closed loops in this representation is $2 \cos (\pi /(p+1))$ (note the shift in the denominator from the weight of the loops in the completely packed lattice model). It is very natural to think of these world lines as domain walls in the $2 \mathrm{~d}$ classical picture, and this $S$ matrix indeed describes the loop model in its dilute phase. Off the critical point, these domain walls have an effective weight per unit length. 
The coupled loop models can be discussed in the same $S$ matrix picture. In fact, it is no more difficult to treat the more general case

$$
\frac{S U(2)_{k} \times S U(2)_{l}}{S U(2)_{k+l}}
$$

which will be discussed in more detail in the next section. This conformal field theory is integrable when perturbed by the (1,1;adjoint) operator (the analog of $\phi_{1,3}$ in the minimal models) of dimension $2(1-1 /(k+l+2))$. This integrable field theory describes the lattice height model of [35] near the critical point, along the line of couplings mentioned above. The $S$ matrices for this field theory have the interesting property that they decompose into the tensor product of $S$ matrices of the minimal models $\mathcal{S}_{k} \times \mathcal{S}_{l}$ [39. Each of $\mathcal{S}_{k}$ and $\mathcal{S}_{l}$ involves a single Temperley-Lieb algebra, so their tensor product can be written in terms of tensor products of Temperley-Lieb generators, namely $1 \otimes 1, e \otimes f, 1 \otimes f$, and $e \otimes 1$. The loop representation of these is exactly that pictured in (3) above! Thus, the heuristic interpretation of this $S$ matrix is that it describes a loop model exactly like ours. Since the $S$ matrix is a tensor product, each particle has structure in both Temperley-Lieb algebras, so its worldlines must be described as doubled lines, and the lines of the different copies can cross at a vertex.

Still more evidence that the dilute loop model has a critical point comes from another solvable model [40] closely related to our dilute loop model. The models in [40] are based on the "dilute BMW" algebra, generalizing those of [24] with weights (20) to allow heights on adjacent sites to be the same. In the graphical representation, this amounts to relaxing the complete-packing requirement. As we have described above in section 3, the $S O(4) \mathrm{BMW}$ algebra decomposes into the direct product of two Temperley-Lieb algebras. Thus the dilute $S O(4)$ BMW algebra in its loop representations describes the same dilute loop model. To make it integrable, one must tune various couplings, including that of the vertex where the loops go straight across. Unfortunately, the corner transfer matrix computation of these models does not seem to have been done, so the CFT describing this critical point has not yet been identified. However, by using the inversion relation method, one can easily compute a critical exponent at each of the two critical points in this model. At the critical point where the weights favor dilute loops, the critical exponent found corresponds to an operator of dimension $3 /(k+2)$, which indeed is present in the coset model (12). Unfortunately, at the isotropic point some Boltzmann weights are negative (although the corresponding $1+1$ dimensional quantum Hamiltonian is hermitian), so it is not possible to make a precise comparison with our dilute loop model.

All this is compelling evidence that the dilute loop has a critical point with the same conformal field theory description as that of the completely packed model at $\lambda=\lambda_{c}$. Minor modifications in the definition of the the loop model, such as allowing multiple strands on a link, or loops to go straight through a vertex, do not change the conclusion.

There are no marginal operators in this coset conformal field theory describing the dilute and completely packed critical point. Thus there should be a flow in the loop model connecting the two points.

Several compelling pieces of evidence imply that the completely packed critical point is stable, i.e., including dilution is an irrelevant perturbation. It has long been known that dilution is irrelevant in the context of a single Potts model. An initial Coulomb gas-type (and thus nonrigorous) argument [41] was subsequently placed on firm ground through the integrability analysis of the most general model of self-avoiding loops defined on the square lattice that is compatible with an $\mathrm{O}(n)$ symmetry [42, 43]. For a given value of $n \in(-2,2)$, this model admits five distinct integrable points with isotropic weights at the vertices. One is the completely packed model 


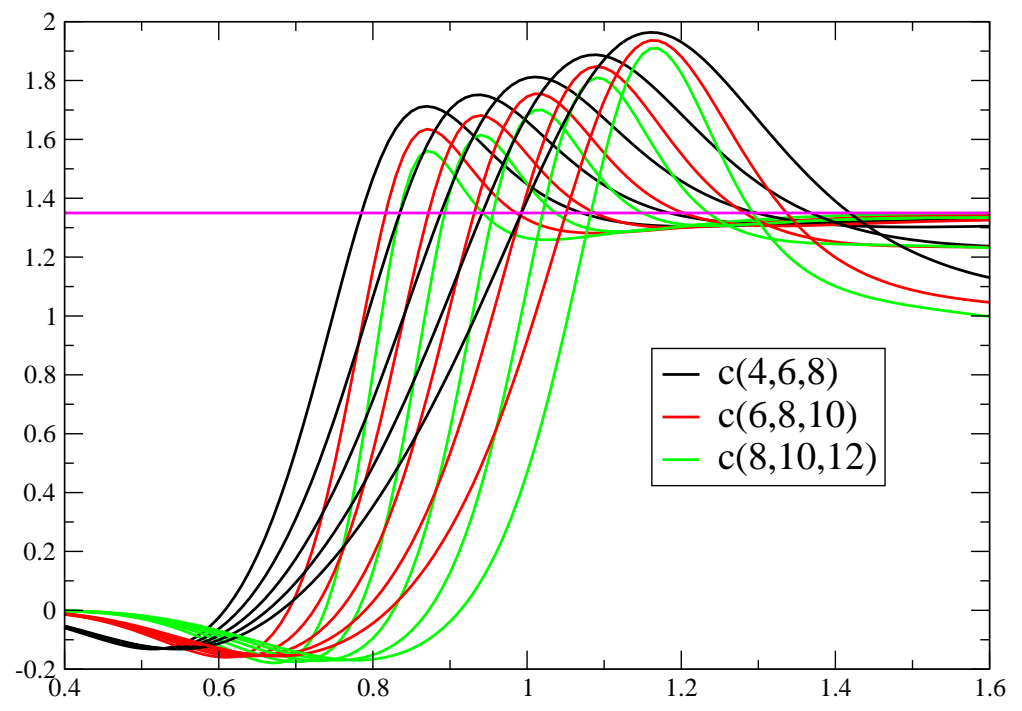

Figure 9: Central charge $c$ as a function of $K$ in the dilute model. The parameter $k=3$, corresponding to $c=\frac{27}{20}=1.35$ (shown as a pink horizontal line) at $\lambda_{c}=-\sqrt{2} \sin \left(\frac{\pi}{20}\right) \simeq-0.2212$. Curves of the same color correspond to several different values of $\lambda=-0.1,-0.2,-0.3,-0.4,-0.5$. As $\lambda$ decreases the curves are shifted towards the right.

which is equivalent to the $Q=n^{2}$ state Potts model. Two other solutions give the so-called dense and dilute solutions for the $\mathrm{O}(n)$ model, and fall in the same universality class [44, 45, as those found previously [46] for the honeycomb lattice [47. Crucially, the dense solution is in the same universality class as the Potts model solution, and so we learn that a deviation from complete packing is an irrelevant perturbation. [The remaining two solutions are multicritical extensions of the dense and dilute solutions.]

Returning to our case of two coupled models, we thus have that at the decoupled critical points at $\lambda= \pm 1$, the flow in $K$ is toward the completely packed line $(K=\infty)$. It is natural to assume that this persists for all $\lambda$, so that in particular dilution is irrelevant both throughout the critical phase discussed in section 4 and near the critical point discussed in section 3.

To complete this picture we turn to numerical transfer matrix calculations. We have been able to study the dilute model (29) for periodic strips of width up to $L=14$ 2 Results for the central charge $c$ as a function of $K$, but fixed $\lambda$, are shown in Fig. 9, still for the case $k=3$. Similar results hold true for other parameter values $k>2$. The various curves correspond to different choices of $\lambda$. The reader may train his/her eye to view this as a three-dimensional plot of $c$ as a function of the two parameters $(K, \lambda)$. Applying Zamolodchikov's c-theorem-which states that

\footnotetext{
${ }^{2}$ Note that the exclusion of straight-going vertices from Fig. 8 is very convenient, since it reduces the dimension of the Hilbert space underlying the transfer matrix construction. Indeed, each element of the space is a pair (one for each loop species) of non-perfect matchings of $L$ points, subject to the usual constraint of planarity with respect to each loop species. In the absence of straight-going vertices, each element in the matching is a pair of points on sublattices of opposite parity. With straight-going vertices allowed, there is no such parity constraint.
} 


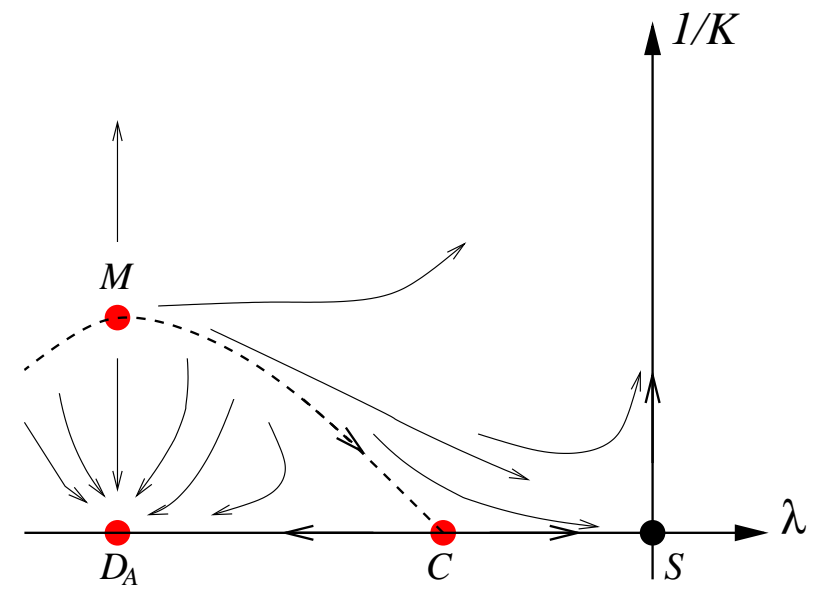

Figure 10: Schematic phase diagram for the dilute model with $k>2$.

RG flows go downhill - reveals that for a large enough $K$ the flow is towards $(K, \lambda)=\left(\infty, \lambda_{c}\right)$, i.e., the critical point of the completely packed model. By "large enough" is meant that $K>K_{c}(\lambda)$, where $K_{c}(\lambda)$ is the locus of the global maximum in $c$. On the other side of the phase boundary, $K<K_{c}(\lambda)$, the flow is towards a non-critical phase, e.g. the trivial fixed point where all loops are absent, or the $(K, \lambda)=(\infty, 0)$ single Potts model point $S$. It is readily verified that dilution is also irrelevant throughout the critical phase. Simulations on the line $\lambda=0$ show that for $k>2$ dilution decreases the effective $c$, so the flow is towards $K=0$.

We therefore arrive at the schematic phase diagram depicted in Fig. 10. The RG flows are shown as arrows. A continuous first-order phase transition occurs along the dashed line. The points $S($ at $\lambda=0), C$ (at $\left.\lambda=\lambda_{c}\right)$ and $D_{A}($ at $\lambda=-1)$ are the same as in the completely packed loop model (see Fig. (4). The point $M$ is a putative multicritical point, unstable in both parameters. For this precise lattice model, we do not know if there is an dilute integrable point like in the lattice models in the same universality class discussed above. If there is, it would be somewhere along the dashed line. Note that there should be an RG separatrix joining $M$ and $S$, as shown.

We have not been able to identify $M$ exactly using our numerical scheme. Because of the $\lambda \mapsto 1 / \lambda$ duality, it is natural to assume that $M$ is located on the decoupling line $\lambda=-1$, but the interference with the first-order transition line impedes the numerical verification of this expectation. Also, determining where the various phase boundaries end, or coalesce, is not an easy matter. We have however verified that the dilute critical phase extending from $C$ is also stable towards a perturbation in the hitherto ignored parameter $\beta$ of (28).

If we generalize the coupled dilute model to include all the interactions present in the most general $\mathrm{O}(n)$ symmetric loop model referred to above [42, 43] we certainly have three candidate multicritical points along the decoupling line $\lambda=-1$ (viz. dilute, multicritical dense, and multicritical dilute). Our constraints on the parameter space are however likely to make at least two of these points disappear. On the other hand, moving away from $\lambda=-1$ could in principle lead to a different kind of multicriticality. 


\section{The self-dual critical point for $Q_{1} \neq Q_{2}$}

At the beginning of section [5, we reviewed the arguments of [17] indicating that the dilute coupled loop models had a critical point. Both the $S$ matrix and the corner transfer matrix arguments apply to a more general class of coset models, those built on the cosets (30). The central charge of this critical point is

$$
c=\frac{3 k l(4+k+l)}{(2+k)(2+l)(2+k+l)}
$$

In the loop language the parameters $k$ and $l$ parameterize the weights for the two different species of loops:

$$
\sqrt{Q_{k}}=2 \cos \left(\frac{\pi}{k+2}\right) \quad \text { and } \quad \sqrt{Q_{l}}=2 \cos \left(\frac{\pi}{l+2}\right) .
$$

These correspond simply to replacing (29) by

$$
Z=\sum_{\mathcal{L}} \sum_{\mathcal{M}} K^{N_{\mathrm{m}}} Q_{k}^{n_{\mathcal{L}} / 2} Q_{l}^{n_{\mathcal{M}} / 2} \lambda^{n_{X}}
$$

so that we are now coupling a $Q_{k}$-state and a $Q_{l}$-state Potts model.

It is thus natural to assume that this loop model with $k \neq l$ will have the same phase diagram as that established above for $k=l$. In particular, there should be a non-trivial critical point in the completely packed model at some negative value of $\lambda$, described in the continuum limit by the coset conformal field theory (30).

We have checked this numerically for several integer values of the parameters $k$ and $l$. Based on the maxima of the central charge (cf. Fig. 55), we find in particular

$$
\begin{array}{ll}
c=1.4675(4) \text { at } \lambda_{c}=-0.2908(7) & \text { for }(k, l)=(3,4) \\
c=1.5431(3) \text { at } \lambda_{c}=-0.3376(2) & \text { for }(k, l)=(3,5)
\end{array}
$$

This agrees nicely with (31) which predicts $c=\frac{22}{15}=1.46667$ for $(k, l)=(3,4)$ and $c=\frac{54}{35}=$ 1.54286 for $(k, l)=(3,5)$. We have unfortunately not been able to provide a convincing conjecture for $\lambda_{c}$ to generalize the exact result (11).

The research of PF has been supported by the NSF under grants DMR/MSPA-0704666 and DMR-0412956, and by an EPSRC grant EP/F008880/1. The research of JLJ has been supported by the European Community Network ENRAGE (grant MRTN-CT-2004-005616) and by the Agence Nationale de la Recherche (grant ANR-06-BLAN-0124-03). The authors thank the Isaac Newton Institute for Mathematical Sciences, where part of this work was done, for hospitality.

\section{References}

[1] H. Temperley and E.H. Lieb, Proc. Roy. Soc. (London) A322, 251 (1971).

[2] See e.g. P.A. Pearce, J. Rasmussen and J.-B. Zuber, J. Stat. Mech. P0611017 (2006) arXiv:hep-th/0607232.

[3] See e.g. J.L. Cardy, J. Phys. A 40, 1427 (2007) arXiv:math-ph/0610030 
[4] I.A. Gruzberg, A.W.W. Ludwig and N. Read, Phys. Rev. Lett. 82, 4524 (1999) arXiv:cond-mat/9902063.

[5] P. Fendley and E. Fradkin, Phys. Rev. B 72, 024412 (2005) arXiv:cond-mat/0502071.

[6] P. Fendley, "Deconfined anyons from quantum loops and nets", to appear shortly.

[7] M.H. Freedman, Comm. Math. Phys 234, 129 (2003) arXiv:quant-ph/0110060.

[8] A.W.W. Ludwig and J.L. Cardy, Nucl. Phys. B 285, 687 (1987).

[9] Vl.S. Dotsenko, J.L. Jacobsen, M.-A. Lewis and M. Picco, Nucl. Phys. B 546, 505 (1999) arXiv:cond-mat/9812227].

[10] J.L. Jacobsen and J. Kondev, Nucl. Phys. B 532, 635 (1998) arXiv:cond-mat/9804048.

[11] R.B. Potts, Proc. Cambridge Phil. Soc. 48, 106 (1952).

[12] R.J. Baxter, J. Phys. C 6, L445 (1973).

[13] G.E. Andrews, R.J. Baxter and P.J. Forrester, J. Stat. Phys. 35, 193 (1984).

[14] D.A. Huse, Phys. Rev. B 30, 3908 (1984).

[15] V. Pasquier, J. Phys. A 20, 1229 (1987).

[16] V. Pasquier, Nucl. Phys. B 285, 162 (1987).

[17] P. Fendley, J. Phys. A 39, 15445 (2006) arXiv:cond-mat/0609435.

[18] R.J. Baxter, Exactly Solved Models in Statistical Mechanics (Academic, London, 1982).

[19] H. Saleur, Nucl. Phys. B 360, 219 (1991).

[20] C.M. Fortuin and P.W. Kasteleyn, Physica 57, 536 (1972).

[21] I. Vaysburd, Nucl. Phys. B 446, 387 (1995) arXiv:hep-th/9503070.

[22] P. Goddard, A. Kent and D. Olive, Phys. Lett. B 152, 88 (1985); Int. J. Mod. Phys. A 1, 303 (1986).

[23] J. Murakami, Osaka J. Math. 24, 745 (1987); J. Birman and H. Wenzl, Trans. Amer. Math. Soc. 313, 239 (1989).

[24] M. Jimbo, T. Miwa and M. Okado, Comm. Math. Phys.116, 507 (1988).

[25] M. Wadati, T. Deguchi and Y. Akustu, Phys. Rep. 180, 247 (1989).

[26] P. Fendley and N. Read, J. Phys. A 35, 1067 (2003) arXiv:hep-th/0207176.

[27] P. Fendley and V. Krushkal, "Tutte chromatic identities from the Temperley-Lieb algebra", arXiv:0711.0016; "Link invariants, the chromatic polynomial and the Potts model", to appear shortly.

[28] A. Kuniba, T. Nakanishi, J. Suzuki, Int. J. Mod. Phys. A 9, 5215 (1994) arXiv:hep-th/9309137]; 5267 arXiv:hep-th/9310060]; 
[29] D. Altschuler, Nucl. Phys. B 313, 293 (1989).

[30] B. Nienhuis in Phase Transitions and Critical Phenomena, ed. by C. Domb and J. Lebowitz, vol. 11 (Academic Press, 1987).

[31] J.L. Jacobsen and J. Salas, Nucl. Phys. B 783, 238 (2007) [arXiv: cond-mat/0703228].

[32] J.L. Jacobsen, J. Salas and A.D. Sokal, J. Stat. Phys. 119, 1153 (2005) [arXiv: cond-mat/0401026.

[33] J.L. Jacobsen and H. Saleur, Nucl. Phys. B 743, 207 (2006) arXiv:cond-mat/0512058.

[34] Y. Ikhlef, J.L. Jacobsen and H. Saleur, Nucl. Phys. B 789, 483 (2008) arXiv:cond-mat/0612037.

[35] E. Date, M. Jimbo, T. Miwa and M. Okado, Lett. Math. Phys. 12, 209 (1986) [Erratum-ibid. 14, 97 (1987)].

[36] E. Date, M. Jimbo, A. Kuniba, T. Miwa and M. Okado, Nucl. Phys. B 290, 231 (1987).

[37] A.B. Zamolodchikov, Mod. Phys. Lett. 6, 1807 (1991).

[38] F.A. Smirnov, Phys. Lett. B 275, 109 (1992).

[39] A. B. Zamolodchikov, Nucl. Phys. B 366, 122 (1991).

[40] U. Grimm, Lett. Math. Phys. 32, 183 (1994) arXiv:hep-th/9402094; U. Grimm and S. O. Warnaar, Nucl. Phys. B 435, 482 (1995) arXiv:hep-th/9407046.

[41] B. Nienhuis, J. Phys. A 15, 199 (1982).

[42] H.W.J. Blöte and B. Nienhuis, J. Phys. A 22, 1415 (1989).

[43] B. Nienhuis, Int. J. Mod. Phys. B 4, 929 (1990).

[44] S.O. Warnaar and B. Nienhuis, J. Phys. A 26, 2301 (1993) arXiv:hep-th/9301026

[45] S.O. Warnaar, P.A. Pearce, K.A. Seaton and B. Nienhuis, J. Stat. Phys. 74, 469 (1994) arXiv:hep-th/9305134.

[46] B. Nienhuis, Phys. Rev. Lett. 49, 1062 (1982).

[47] M.T. Batchelor and H.W.J. Blöte, Phys. Rev. Lett. 61, 138 (1988). 